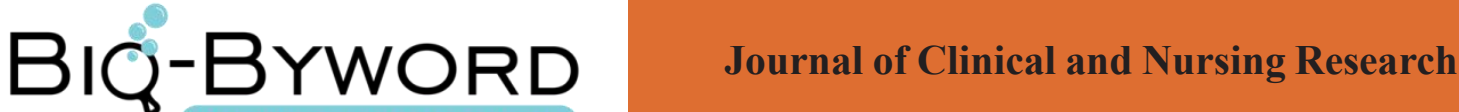

\section{Evaluation of Nursing Effect of Comprehensive Nursing Intervention on Elderly Patients with Severe Pneumonia}

\author{
Chen Hua Shuang
}

Chengdu Elderly Home, Donghong Elderly Hospital, Intensive Care Unit, Sichuan Chengdu 610058

\section{ARTICLE INFO}

Article history:

Published online: $30^{\text {th }}$ Sept, 2017

\section{Key words:}

Comprehensive nursing intervention

Elderly patients with severe pneumonia

Nursing effect

\begin{abstract}
Objective: To analyse and discuss the related aspects of the nursing effect of comprehensive nursing intervention in elderly patients with severe pneumonia. Methods: 150 cases of senile severe pneumonia were randomly divided into observation group and control group. 75 patients were distributed to each control group and observation group, the control group was applied with conventional nursing intervention, observation group was applied with comprehensive nursing intervention, the two groups of clinical efficacy and quality of life and related care satisfaction were compared. Results: The effective rate of treatment was $90.67 \%(68 / 75)$ in the observation group, which was significantly higher than that in the con-trol group $(74.67 \%, 56 / 75), \mathrm{P}<0.05$; the observation group's body function and mood and role, social function $(\mathrm{P}<0.05)$ were significantly higher than that in the control group. The satisfaction rate of the observation group was $96.00 \%$ (72/75), which was significantly higher than that of the control group (82.67\%) (62/75), $\mathrm{P}<0.05$. Conclusion: For elderly patients with severe pneumonia, the comprehensive intervention care is obviously having better effect. By having comprehensive intervention care, the clinical treatment effect and nursing satisfaction can be improved in a certain sense, and improved the quality of life of the patients.
\end{abstract}

\section{Introduction}

Severe pneumonia cause high mortality, causing a serious threat to patient's life. Severe pneumonia disease progress very fast, leading the occurrence of pathogens like haemolytic streptococcus, streptococcus pneumonia and so on. For elderly patients, they have lower immunity, and their organ undergo varying degrees of degradation, and most of their body suffering chronic diseases, resulting more prone to severe pneumonia. In order to ensure the therapeutic effect and improve the prognosis of patients, scientific and reasonable nursing intervention is required in the course of clinical treatment. How to effectively care for elderly patients with severe pneumonia is a key concern. This study evaluates the effect of comprehensive nursing intervention in elderly patients with severe pneumonia and provides a reference for the development of nursing interventions for elderly patients with severe pneumonia.

\section{Materials and Methods}

\subsection{General Information}

150 cases of severe pneumonia in our hospital from May 2015 to May 2017 were selected as the research object, which were in accordance with the diagnostic criteria of severe pneumonia developed by the 2006 Chinese Medical Association Respiratory Diseases Branch. They were randomly divided into observation group ( 75 cases) and control group (75 cases). The observation group was 41 males and 34 females; aged 65-83 years, mean (69.13 \pm 5.46 ) years old; 26 cases of right lung lobe, 8 cases of left lower lobe, 11 cases of right lower lobe, 7 cases of lung infection; combined with basic disease: chronic 
obstructive pulmonary disease in 17 cases, 12 cases of pulmonary abscess, bronchial dilatation in 13 cases The control group: 75 cases, 42 males and 33 females; aged 65 to 83 years, mean $(70.25 \pm 5.38)$ years; lung infection: 24 cases of the middle right lung, 26 cases of right upper lung, 11 cases of right lower lobe, lung infection in 5 cases; combined with underlying disease: 16 cases of chronic obstructive pulmonary disease, pulmonary abscess in 12 cases, 15 cases of bronchiectasis. Approved by the Hospital Ethics Committee, all patients informed consent.

\subsection{Methods}

Two groups of patients were admitted to the hospital after the routine treatment, including persistent low-flow oxygen, cardiac, sedation, expectorant, asthma, nutritional support, to correct water and electrolyte disorders, to maintain acid-base balance and antibiotic anti-infective treatment. Usually in the actual care, the acute phase should be bed rest, especially for patients who have not yet returned to normal body temperature. Bed rest can reduce tissue oxygen consumption and contribute to body tissue repair. In order to help patients lie halfway, increase ventilation and reduce breathing difficulties. Keep the indoor air fresh, sunny, neat, quiet and comfortable, and limit the visit. Room temperature should be maintained at 18-20, humidity $50-60 \%$ to prevent air become too dry, dry air reduce tracheal ciliary motor function, resulting poor sputum. High fever decreased digestion and absorption capacity, the body catabolism increased, carbohydrates, protein, fat and vitamins and other nutrients consumption increased, should be given high calorie, high protein, rich in vitamins, light digestible or semi-liquid diet. Encourage patients to drink plenty of water, the daily intake should be more than $2000 \mathrm{ml}$. High fever patients need intravenous fluid, but should pay attention to control the drip rate, to prevent causing pulmonary edema. Encourage physical exercise, so that patients can withstand a certain amount of physical activity and exercise, improve the respiratory function, to maintain normal gas exchange. We can guide the elderly patient exercise, such as: gymnastics, tai chi, walking, etc., help to improve the body resistance, enhance disease resistance. In this case, the control group using conventional nursing intervention, the observation group using comprehensive nursing intervention, specifically the following related aspects of the content:

\subsubsection{Observe the Patient's condition}

Closely observe the elderly patients with severe pneumonia in general, such as vital signs and the indicators. The patient's condition to conduct a comprehensive assessment of the risk factors may occur to exclude the indicators, such as whether hypoxemia, with or without high carbon dioxide and so on. Ventilation dysfunction, the body hypoxia occurs, the patient may have respiratory symptoms such as changes in symptoms, so in daily care, should pay attention to whether the abnormal breathing frequency. The main manifestations of septic shock for blood pressure, body temperature decreased, so patients with blood pressure, body temperature should be considered whether the decline due to septic shock. Patients with severe brain tissue hypoxia, ischemia or the occurrence of carbon dioxide retention, the general performance of the early abnormalities. Therefore, in the early detection of patients with changes in condition, should be promptly reported to the attending physician, and as soon as possible to deal with to prevent the occurrence of critical illness.

\subsubsection{Respiratory tract Care}

In the treatment of elderly patients with severe pneumonia in patients with humidification respirator treatment process, the respiratory tract care is also important. Respiratory tract management mainly includes the following four parts, namely, continuous respiratory tract humidification, drug ultrasonic atomization, auxiliary shot back expectoration, effective sputum suction and so on.

First, the continuous respiratory tract wetting process. Due to infection and other factors caused by severe pneumonia in elderly patients with a series of respiratory symptoms, such as viscous secretions; weakened breathing or tracheal mucosal ciliary movement stopped; sputum difficult to cough out or even the formation of sputum bolt; due to respiratory tract, blocked causing difficulty in breathing; lips cyanosis and so on. The application of humidifier enhanced the patient's respiratory tract clearance ability, and help to cure patient in certain degree at the same time. In the clinical practice, humidifier gradually replaced the conventional way of humidification, due to simple operation, highly effective features. Nursing process should pay attention to the following:

(1) correct connection of piping and equipment. (2)Using the right humidifying solution, add appropriate amount of humidifying solution in the humidifier with sterile water for injection. The infusion tube is connected to the humidification tank, and connected to the humidifying solution too. When the gas temperature and the gas flow sent out, the self-generated negative pressure control humidifying solution dropped into the wetting tank in uniform speed, to let the liquid in the tank reach the optimum volume. (3) Set the correct value of gas flow parameter, as to let the positive pressure flow of the respiratory tract can be maintain, the tidal volume of the patient is significantly increased, the breathing frequency is significantly reduced and the dyspnoea problem can be improved. Set the effective temperature parameter, enhance the patient's cilia cleaning function, resulting a cleaned respiratory tract. (4) Humidifying effect judgment and setting of treatment time, the indication of respiratory tract humidifying is sputum viscosity, the sputum viscosity is divided into three grades, level one: thin sputum, like rice soup, easy to cough out; level two: slightly viscous sputum, can cough out if cough harder; 
level three: viscous sputum, yellow in color, sticked to the trachea wall, it is difficult to be cough out. Level one sputum patients, suggest 4-6 hours of continuous humidifying breathing treatment, 2-3 times/day; level two sputum patients, the suggested time is $8-12$ hours of continuous humidifying breathing treatment, 1-2 times/day; level three sputum patients, it is recommended to continue to humidifying breathing treatment for 24 hours. Clinical practice found that most of the level three sputum patients had became level two or level one sputum after one-week of treatment, the patient's clinical Second, patting patient's back to help expectoration. As the patient's ability to expectoration are poor, patient often need help in expectoration, auxiliary sputum, including two ways, and machine expectoration and manually expectoration. Before helping expectoration, we should assess the patient's tolerance level according to the patient's condition, then set the appropriate intensity and frequency. The new type of chest percussion method is currently commonly used in the percussion techniques, the specific operation process is as follows, the patient was lateral position, nursing staff standing on the back of the patient, the palm was making like a cup shape, in the patient's thoracic part from the outside, the use of The strength of the wrist of the wrist of the back of the patient, the adjacent tapping part of the need for $1 / 3$ of the overlap, in the side of the percussion is completed, tapping the other side, 10-15 minutes/time, 2-3 times/day.

Third, sputum suction. The key link of respiratory tract care is the effectiveness of sputum discharge. During the operation of suctioning, the effectiveness of sputum suctioning is judged. The process of suctioning is required to be aseptic. Therefore need to be careful to avoid injure to mucosa. Continuous suction should be maintained within three times, and according to the patient's sputum situation, adjust the daily suction frequency. Cough up the sputum difficult patients to ensure that every two hours once, after the patient's symptoms are reduced, gradually reduce the frequency of suction.

\subsubsection{Environmental Care}

Must ensure that the ward is comfortable, quiet, keep the air fresh, the temperature and humidity must be appropriate, and window ventilation $>3$ times/day, while taking ultraviolet light 2 times/day, irradiation time 30 min/times, or daily with air disinfection 2 times, each time 1 hour; at the same time the number of visits to patients and time to give strict restrictions, so as to avoid cross-infection.

\subsubsection{Psychological Care}

Because the patient is in critical condition and is prone to a variety of negative emotions in the clinic, the clinical care provider must give the patient a health education manual that allows the patient to understand their quality, purpose, function, and related precautions. So that the patient's cardiac load and myocardial oxygen symptoms improved significantly, respiratory tract cleanliness has also increased. (5) Closely monitor the changes of patient's condition, in line with the removal criteria, start using humidifier for treatment, in its application period, check the patient's blood gas analysis in schedule, emergency drug medicine and first aid equipments should be standby. (6) Sterile isolation, during humidifier treatment process, sterile isolation should be done on the instrument, instrument function should be check once a month.

consumption was significantly reduced.

\subsubsection{Medication Care}

In the establishment of intravenous access to patients, the clinical nurses to skilful operation skills to reduce the pain caused by the puncture to patients, according to the nature of the drug and the use of drugs to choose a reasonable

injection site, in patients with anti-fever drugs, the patient to use drugs, and give close observation.

\subsubsection{Oral Care}

The bacteria in the patient's mouth will move down along the trachea, so the clinical care workers should strictly do oral care. If the $\mathrm{pH}$ value is $1.0-7.5$, you can take $2 \%$ to $3 \%$ of the boric acid Solution. General patients will be associated with emphysema, the main symptoms of dyspnoea, this time should take oxygen therapy, to maintain oxygen flow of $1-5 \mathrm{~L} / \mathrm{min}$, at the same time, clinical care workers also close observation of patients with hypoxia, after the disappearance of cyanosis in patients with oxygen.

\subsubsection{Skin and Sleep Care}

Older patients with severe pneumonia in poor physical condition, the daily stay in bed longer, plus some patients do not form a good health habits, so they prone to pressure sores, skin infections and other complications, which will affect the rehabilitation of their bodies. In response to this situation, the caregiver should inform the patient's family regularly for the patient to clean the skin, every $2 \mathrm{~h}$ for its turn 1 , and massage the long-term compression of the site. In addition, the elderly are more sensitive to the external environment, so every night when they fall asleep, nurses should pay attention to keep the ward environment quiet, adjust the air conditioning temperature, and cover the quilt, to prevent the occurrence of a cold.

\subsubsection{Health Education}

Clinical care personnel to patiently explain their causes of morbidity and improve the prevention of self-awareness of patients, at the same time, clinical care workers to correctly guide patients to increase the amount of drinking water, easy to digest food, banned eating cold, 
spicy food, and clearly inform the patient, most important to quit alcohol and tobacco, the proper guidance of patients after discharge to take appropriate aerobic exercise, timely vaccination of pneumonia.

\subsubsection{Other Precautions}

Due to the particularity of old age pneumonia, nurses should pay attention to the early detection of early pneumonia in elderly patients, early diagnosis and prevention, reduce morbidity and mortality. Winter and spring for the elderly pneumonia season, the incidence and the incidence of respiratory virus infection. Older pneumonia patients with mild upper respiratory tract infection, after the bacteria in the alveoli infection. Elderly patients should avoid the rain and catch cold, fatigue, alcoholism, mental stimulation, viral infection and other predisposing factors. When the weather changes, should be added to the appropriate clothing, to avoid public places, if necessary, can wear a mask, other family members suffering from influenza, can use vinegar to fumigation room. Daily exercise, enhance physical fitness. From the summer began to adhere to cold water wash, more contact with fresh air. Long-term adherence, can improve the body's tolerance to the cold, winter respiratory tract infection will be greatly reduced. Bedridden elderly patients turn over to prevent the occurrence of hypostatic pneumonia. Elderly patients are prone to immune dysfunction and other diseases. Should actively treat their diseases, take effective measures to improve the immune function of elderly patients,

but also vaccination against pneumonia to prevent pneumonia. In elderly patients with pneumonia onset conceal, often no obvious cough, sputum, chest pain, fever and other symptoms, therefore, elderly patients with non-respiratory symptoms serious treatment should pay attention to whether there should be pneumonia, conventional X-ray or chest examination in the disease early diagnosis.

\subsection{Observe Indicators}

Observe the efficacy of the two groups of treatment, efficacy: (1) Markedly: the patient's sputum, cough and other clinical symptoms were significantly alleviated or disappeared, significantly improved arterial blood gas parameters, lung X-ray examination returned to normal; (2) Effective: patients Of the sputum, cough and other clinical symptoms have been a certain degree of relief, arterial blood gas parameters partially improved; (3) Invalid: patients with sputum, cough and other clinical symptoms were not improved or even worse, the condition deteriorated or died. The nursing satisfaction and quality of life of the two groups were compared. The quality of life (physical function, emotional function, role function and social function) of the two groups were assessed by the Health Status Questionnaire (SF-36). The higher the score, the higher the quality of life. The satisfaction survey of the patients was conducted by the self-designed questionnaire, which scored 60 points or less expressed dissatisfaction, score 60 - 84 points expressed satisfaction, 85 points or more is very satisfied.

\subsection{Statistical Analysis}

SPSS 15.0 software was used to measure the data with $\mathrm{x}$ $\pm \mathrm{s}$. Compared with $\mathrm{t}$ test group, the difference was statistically significant by comparison with the $\chi 2$ test.

\section{Results}

\subsection{Comparison of Two Groups of Clinical Efficacy}

The effective rate of the observation group was $90.67 \%$ $(68 / 75)$, which was significantly higher than that of the control group $(74.67 \%, 56 / 75)(\mathrm{P}<0.05)$. Please refer to table 1 .

Table 1 Comparison of two groups of clinical efficacy

\begin{tabular}{cccccc}
\hline Group & $\mathrm{n}$ & Less effective & Effective & Not effective & Effective rate \\
\hline Observation Group & 75 & $31(41.33)$ & $37(49.33)$ & $7(9.33)$ & $90.67^{*}$ \\
Control Group & 75 & $23(30.67)$ & $33(44.00)$ & $19(25.33)$ & 74.67 \\
\hline
\end{tabular}

\subsection{Comparison of Two Groups of Quality of Life Scores}

The physical function, emotional function, role function and social function score of the observation group were significantly higher than those of the control group $(\mathrm{P}<0.05)$. Please refer to table 2 .

Table 2 Comparison of two groups of quality of life scores

\begin{tabular}{cccccc}
\hline Group & $\mathrm{n}$ & Physical funciton & Emotional function & Roles function & Social function \\
\hline Observation Group & 75 & $1.99 \pm 0.73^{*}$ & $1.87 \pm 0.62^{*}$ & $1.78 \pm 0.59^{*}$ & $1.16 \pm 0.43^{*}$ \\
Control Group & 75 & $1.42 \pm 0.65$ & $1.34 \pm 0.57$ & $1.22 \pm 0.43$ & $0.70 \pm 0.23$ \\
\hline
\end{tabular}

\subsection{Comparison of Two Groups of Nursing Satisfaction}


The care satisfaction of the observation group was $96.00 \%(72 / 75)$, which was significantly higher than that of the control group $(82.67 \%, 62 / 75), \mathrm{P}<0.05$. Please refer to the table 3 .

Table 3 Comparison of two groups of nursing satisfaction

\begin{tabular}{cccccc}
\hline Group & $\mathrm{n}$ & Very satisfied & Satisfied & Less satisfied & Satisfied rate \\
\hline Observation Group & 75 & $51(68.00)$ & $21(28.00)$ & $3(4.00)$ & $96.00^{*}$ \\
Control Group & 75 & $40(53.33)$ & $22(29.33)$ & $13(17.33)$ & 82.67 \\
\hline
\end{tabular}

\section{Discussion}

Severe pneumonia in addition to pneumonia which is the most common respiratory symptoms, often accompanied by systemic symptoms of poisoning, while accumulating other systems caused by complications, the sudden onset, critically ill, rapid progress, mortality is relatively high, the relative difficulty of clinical treatment bigger. According to the study shows that in patients with severe pneumonia to take a positive and effective treatment based on a comprehensive nursing intervention, can make the patient's symptoms and efficacy to achieve satisfactory results. In addition, according to the study shows that patients with severe pneumonia, the purpose of taking comprehensive care intervention is to be able to effectively relieve the patient's lung function and psychological and other related issues, most of the psychological aspects of fear and anxiety, so clinical nursing staff in the implementation of nursing intervention when patients must be on their physical and mental conditions have a clear understanding, while taking a comprehensive nursing intervention, not only to enable patients to maintain physical and mental pleasure, while allowing patients to take the initiative to cooperate with clinical treatment, Disease significantly reduced, the quality of life of patients play a positive role in promoting.

Routine care is more common in clinical practice, but the nursing measures have a certain randomness and one-sidedness. Nursing staff only to patients with routine disease monitoring, to maintain the ward environment, although to some extent control the occurrence of complications, but the caregivers did not focus on the prevention of complications and physical condition of the patient to improve the implementation of effective care measures, while patients The impact of disease symptoms prone to anxiety, psychological fear, nursing staff did not focus on the psychological mood of patients with psychological counselling measures, but the patient's compliance and the impact of the disease effect.

Comprehensive care is a comprehensive assessment of nursing care and analysis of elderly patients with severe pneumonia, combined with the relevant medical knowledge, timely detection of nursing process potential deficiencies, and improve the patient for the implementation of comprehensive care measures, the presence of care risks of effective prevention. Nurses in the disease treatment and care in all aspects, according to the law of disease development, physiological basis and symptoms, and actively take the patient to implement the appropriate nursing measures, comprehensive treatment of the disease may occur in the process of adverse events thereby enhancing the quality of life of patients. Comprehensive care mainly through the care of patients with a comprehensive and scientific analysis of the disease, and for the development of a comprehensive care program to guide and encourage patients to effectively take deep breathing and sputum, for the sputum is more viscous and cough more difficult large patients to implement inhalation, so that the smooth discharge of sputum, to avoid infection caused by bacterial infection, thereby reducing the occurrence of patients with dyspnoea and pulmonary dysfunction; through the implementation of health education for patients and their families and psychological counselling, is conducive to enhance the family's awareness of the disease, but also to improve the psychological mood of patients to establish the confidence of the treatment of disease; nurses by closely observing the patient's condition changes, their care experience and the actual situation combined with the patient may occur complications predict, and then strengthen the care of patients, in order to reduce the probability of complications in patients.

In the course of clinical treatment of elderly patients with severe pneumonia, care is more difficult, if you can correctly grasp the characteristics of the patient's disease, to give effective care intervention, can effectively improve the clinical treatment of patients. Diversification of nursing interventions has been clinically proven, and various care interventions have different roles at each stage of the disease. Comprehensive nursing intervention is a kind of quality nursing service which is based on the nursing process and the modern nursing concept, according to the patient's social, physical and psychological and cultural needs. The results showed that the effective rate of the observation group was $90.67 \%$ (68/75), which was significantly higher than that of the control group (74.67\%) (56/75) in the control group, and the observation group was treated with routine nursing intervention. $(\mathrm{P}<0.05)$. The satisfaction rate of the observation group was $96.00 \%(72 / 75)$, which was significantly higher than that of the control group ( $\mathrm{P}<$ 0.05 ), and the physical function, emotional function, function and social function score of the observation group were significantly higher than those of the control group $82.67 \%(62 / 75)$ of the control group, $\mathrm{P}<0.05$. It is suggested that the comprehensive nursing measures such as medical environment, psychological nursing, diet 
nursing and health education can improve the clinical treatment effect and nursing satisfaction, and improve the quality of life of patients with severe pneumonia.

\section{Conclusion}

In summary, comprehensive nursing intervention has significant nursing effect on elderly patients with severe pneumonia, improve the clinical treatment effect and nursing satisfaction, improve the quality of life of patients, have a high clinical value.

\section{References}

[1] Zeng Juan, Wang Yali, Liu Ting. Coronary heart disease intensive care unit anxiety, Evaluation of the effect of comprehensive nursing intervention in depressed patients. Journal of Practical Clinical Medicine, 2017, 21(12): 131-133.

[2] Liu Yuanyuan. ICU severe pneumonia in patients with mechanical ventilation comprehensive nursing intervention and effect observation. Practical Clinical Nursing E-Zine, 2017, 2(08): 22-25.

[3] Zhang Zhenzhen. Comprehensive respiratory care for elderly patients with severe pneumonia in the respiratory tract inflammation and treatment of the effect. System Medicine, 2016, 1(12): 155-157.

[4] Chang Haiying. Severe pneumonia with respiratory failure in patients with nursing experience. Clinical Medicine Journal of Electronic Journal, 2016, 3(40): 7997.

[5] Xu Fang. Effect of comprehensive nursing intervention on ventilator-associated pneumonia in elderly patients. We Health, 2016, 10(12): 230-231.

[6] Wei Yushan, Xiao Qike. Effect of comprehensive nursing intervention on prevention of hypostatic pneumonia in elderly patients after fracture. Medical Equipment, 2016, 29(11): 163-164.

[7] Yan Ping, Chen Fengying, Luo Yinzhen. Effect of comprehensive nursing intervention on prevention of hypoglycaemia after total hip arthroplasty in elderly patients with diabetes mellitus. The World's Latest Medical Information Digest, 2016, 16(28): 292-295.

[8] Jiang Li. The effect of comprehensive nursing intervention on the elderly patients with acute severe cholangitis. Chinese Journal of Practical Medicine, 2016,11 (04): 239-240.

[9] Cao Juan. Nursing intervention in elderly patients with pneumonia. We Health, 2015, 9 (23): 247-248.

[10] Li Xuefeng, Hu Chunhuan, Lu Jie. Evaluation of the effect of comprehensive nursing intervention on children with severe pneumonia. Journal of Health Medicine Research and Practice, 2015, 12(05): 83-88.

[11] Shao Jihong, Wu Yiqun, Zhang Peifu. Effect of Comprehensive Nursing Intervention on Improving Elderly Hepatitis B Cirrhosis with Type 2 Diabetes Mellitus. World Chinese Journal of Digestion, 2015, 23(23): 3771-3774.

[12] Zhang Xinrong. Effect of comprehensive nursing intervention on elderly patients with ventilator-associated pneumonia. Contemporary Medical Papers, 2014, 12(15): 111-112.

[13] Wang Lin. Elderly care ward metabolic syndrome in patients with comprehensive intervention effect evaluation. Chinese Community Physician (Medical), 2012, 14(09): 84-85. 